\title{
Multispectral Palmprint Recognition Based on Multidirectional Transform
}

\author{
B. OZMEN and O. J. OLALEYE
}

\begin{abstract}
Multispectral palmprint recognition is one of the most useful biometric techniques due to features obtained from different spectral resolutions/wavelengths. In this paper, we propose a multidirectional transform-based feature encoding plan for reliable and robust representation and matching of multispectral palm images. The method extracts the region of interest (ROI) for palmprint images captured with non-contact sensors. The registered ROI of each band is newly downsampled using DWT. This approach allows us to take more lines into consideration for interpolation. A undecimated dual-tree complex wavelet transform based multidirectional feature encoding plan is then newly applied since it provides better shift invariance and directional selectivity. Finally, a binary code matching strategy with score level fusion is used to compute matching for efficient identification. The experimental results obtained on CASIA and PolyU datasets show that the presented method gives better results in the blurring binary code matching case than state-of-the-art methods and provides comparable performance in the non-blurring binary code matching.
\end{abstract}

Index Terms-Feature Extraction, Image Recognition, Matching, Multispectral Encoding, Pattern Analysis, Wavelet Transforms.

\section{INTRODUCTION}

$\mathrm{B}$ IOMETRICS IS THE method used to recognize individuals based on one or more authentic physical and/or behavioral traits [1]. Palmprint recognition has attracted much attention from researchers [2]-[6] due to its ease of use, high speed, robustness, reliability and affordability. 3D imaging can be used to improve the accuracy of the palmprint system and ability to detect spoof attacks [7]. However, improper tools and the cost of the systems make it difficult to use. Multispectral imaging can be one solution [8][10]. It captures an image in a range of spectral bands.

B. OZMEN, is with Department of Electrical \& Electronics Engineering, European University of Lefke, Lefke, Northern Cyprus, TR-10 Mersin, Turkey, (e-mail: bozmen @eul.edu.tr).

iD https://orcid.org/0000-0002-3608-394X

O. J. OLALEYE, is with Department of Electrical \& Electronic Engineering, Cyprus International University, Haspolat - Lefkoşa, Northern Cyprus, TR-10 Mersin, Turkey, (e-mail: olaleyeolayinka30@ gmail.com).

iD https://orcid.org/0000-0003-0692-0847

Manuscript received January 28, 2019; accepted April 22, 2019. DOI: $\underline{10.17694 / \text { bajece. } 518050}$
Specific features of each palm can be obtained from different spectral bands so that we have enough information to enhance its accuracy. The antispoofing capability of palmprint systems can be improved.

The unique patterns that can be seen with the naked eye are the principal lines, the fine edges and the wrinkles [11]. Standard imaging devices can capture these superficial features. High-wavelength scanners can capture the fine ridges that can be used for inert palmprint identification in forensics [12]. The wrinkles and principal lines captured with lowwavelength sensors are well-suited for security applications such as client identification or authentication [13]. The subsurface vein pattern is extra information presented in the human palm. The subsurface vein pattern is unrelated to the palm lines. Standard imaging sensors cannot identify such features. Infrared imaging can capture subsurface features due to its capability to penetrate the skin. Multispectral palmprint recognition can be used to investigate the subsurface and superficial features of a palm. Multispectral imaging captures images at multiple wavelengths. These complementary features (veins and palm lines) allow for increased discrimination between persons. These features of palm images are important to improve performance where user cooperation is moderate, such as secure access gates, personality records and workplace entry.

Multispectral palm pictures can be captured by using monochromatic camera under spectrally changing illumination [14], [15]. Hand movement is restricted by contact device but increases user admissibility issues due to hygiene. Nevertheless, biometrics which are captured with non-contact sensors are more useful and socially more sufficient [16], but create the problem of Rotation-Scale-Translation (RST) variations. The movement of the palm is the reason for these misalignments. Landmark detection and ROI extraction must be applied to solve this problem. A robust feature extraction strategy is required for precise recognition of line-like edges, because of the multi-modal nature of the palm. In addition, multi-modal feature extraction results with many features cause slow matching time and require large storage resources. These challenges can be solved with a feature encoding plan and matching technique.

This paper presents a new multidirectional transform-based feature encoding strategy for reliable and robust representation and matching. A reliable method is applied to extract ROI from non-contact palmprint images; a robust directional and multiscale feature encoding method is proposed for multispectral palmprint images; and an effective binary code matching strategy is used for matching process and compact storage of multidirectional components. Presented approach is 
compared with state-of-the-art methods by using different experimental setups.

\section{RELATED WORKS}

Using multispectral images, biometrics like the iris [17], face [18], [19] and fingerprint [20] have been researched for improved precision in the past decade. Researchers have now turned their attention to multispectral palmprint recognition [14], [15], [21]-[32]. Palmprint recognition techniques can be categorized as texture-based coding methodologies, line-like feature finders and subspace learning strategies [33]. The combination of these three classes is possible. Edge detectors can be used to extract palm lines in line detection-based methodologies. A palmprint verification method by utilizing principal lines is presented in [34]. The principal palm lines were separated by using a modified finite Radon transform. However, recognition based on only palm lines was inadequate [27]. Palm lines can be extracted by line detection. However, line detection is not suitable for extraction of palm veins due to their broad structure and low contrast.

The global characteristics of the palm can be captured by using subspace projection. Subspace projection strategies incorporate Eigen-palm [35], which reflects palm images to a PCA space, or Fisher-palm [36] which reflects to a LDA space. However, such subspace projection is not well suited to preserve and model finer local details. In [37], Laplacian palm representation is proposed by fusing palmvein and palmprint images. In that work, Laplacian palm representation is used to preserve local characteristics while reflecting onto subspace unlike Eigen-palm [35] and Fisher-palm [36]. Multispectral palmprint images are represented as quaternion PCA and employed quaternion PCA to get features. For recognition, a nearest neighbor classifier is used. The quaternion model was not as effective as state-of-the-art techniques in representing multispectral palm images and were found to have low recognition accuracy. The subspace learned from incorrect align palms cannot create an exact representation of every identity.

The methods based on orientation encode and extract the directional lines. Those methods have shown state-of-the-art performance in palmprint recognition [11]. The Competitive Code (CompCode) [38], the Ordinal Code (OrdRepr) [39] and the Derivative of Gaussian Code (DoGRepr) [40] can be examples of the orientation-based methods. In the generic form of the methods based on orientation coding, each directional subband is identical to a certain orientation of lines. The orientation-based code is formed from the dominant orientation index, extracted from each of the directional subbands. A directional bank of Gabor filters is used to extract the orientation of palm lines in CompCode [38]. Coefficients of the directional subbands are encoded into a binary code. Then, the results are matched directly by applying the Hamming distance. The OrdRepr [39] studies the ordinal relationship of lines. This method compares reciprocally orthogonal filter pairs to get feature orientation at a point. The DoGRepr [40] uses vertical and horizontal derivative filters to extract feature orientation. The coefficients of the orientation-based methods can be binarized for effective storage and fast matching compared to other representations that need floating point data storage and calculations. Another critical part of multispectral palmprints is the way to combine the spectral bands. The multispectral palmprints have been examined with feature, data, rank-level combination and score.

Multiresolution transform techniques such as wavelets and curvelets are used to fuse multispectral palmprint images. A three-scale wavelet transform methodology is used for fusion multispectral palmprint images in [14] and then they applied CompCode for feature extracting and matching. The results showed that the wavelet combination of multispectral palm images is valuable for blurred palm images. In [32], different image fusion methods are used. The OLOF was one of those representations for multispectral palmprint recognition. The best recognition accuracy was accomplished when the curvelet was used for band combination. A wavelet-based band combination and a Gabor wavelet-based feature representation for multispectral palm pictures are presented in [28]. Feature selection was done using Ant Colony Optimization (ACO) [41] to reduce the dimensionality and classified by SVM and normalized correlation. However, Gabor wavelet-based fusion did not give better results than the curvelet combination with OLOF [32] for palmprint recognition. A joint palmline strategy is proposed in [27] to have multispectral palmprint recognition. Different feature encoding techniques were designed for palmline and palmvein. Later, score-level fusion was used to compute the final match. This approach showed more promising results than a feature- and/or rank-level fusion. In addition, single palmprint image representation may be extended to multispectral cases. However, features in different bands such as lines and veins might not be preserved [27]. A comparative study presented in [12] showing that local orientation-based features are the recommended option for palmprint image feature extraction.

This paper proposes a new multidirectional transform-based feature extraction considering multispectral palmprint recognition. UDTCWT is used to represent coefficients since it gives superior performance with directionality and shift invariance properties than UDWT and DTCWT. A two-level filtering strategy is presented to obtain more robust and useful multidirectional features than those of orientation-based methods that use a directional filterbank. The idea presented in [42] is used for the binary matching strategy. Three orientation-based techniques [38] [39] [40] are implemented and compared with the presented method considering different experimental setups. The scheme of the presented method is depicted in Fig. 1.

The rest of this paper is organized as follows. In Section III, we explain a solid procedure for ROI extraction from multispectral pictures produced by means of non-contact sensors. The proposed feature extraction method and applied binary code matching and coordinating procedures are discussed in Section IV. In Section V, we introduce a pointby- point trial examination to assess the precision of the multispectral palmprint image analysis. We investigate the impact of different parameters. We perform verification and identification tests in different trial settings using two standard multispectral palmprint databases and compare the proposed 


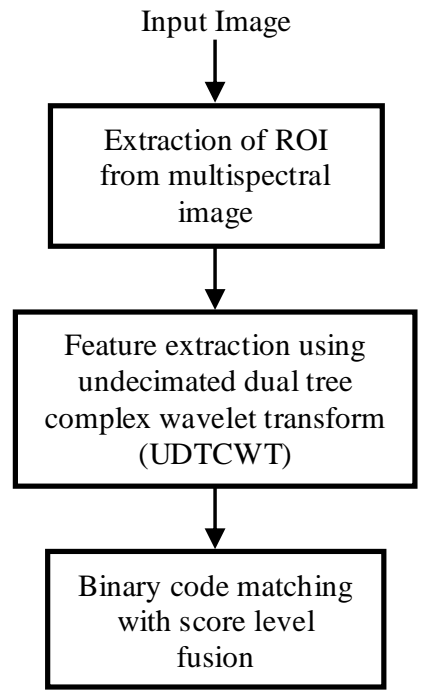

Fig. 1. Schematic diagram for the presented method.

method with the state-of-the-art methods. Conclusions are given in Section VI.

\section{REGION OF INTEREST EXTRACTION}

For feature extraction and matching, it is necessary to extract a specific portion from the palmprint image which is known to be ROI extraction. There are many advantages to applying ROI extraction processes to the images captured from noncontact sensors. One of these advantages is to be used to eliminate translation, scale and rotation of palm vein images. ROI extraction process also extracts the most useful area in the images. It reduces the amount of data without missing much useful information. This process affects the speed and the quality of the feature extraction and matching processes. The ROI extraction method introduced in [42] is used.

Since each band of the multispectral images is sequentially captured, minor hand movements cannot be eliminated. Therefore, the maximization of mutual information explained in [32] is used for inter-band registration of the ROIs. The strategy was effective for registering multispectral palm images. Once the registration of ROIs for each band is completed, it is resized to $128 \times 128$ pixels with bicubic interpolation and then downsampled to $32 \times 32$ pixels using the discrete wavelet transform rather than the method used in [42]. This re-sampling step shows better performance than bicubic interpolation because it reduces the noisy regions and inconsistent lines while taking more lines or points into consideration for interpolation. This approach reduces the amount of required storage in the final feature. The required time and final stage storage are also reduced in case of extraction and matching processes. Process for extraction of ROI is shown in Fig. 2.

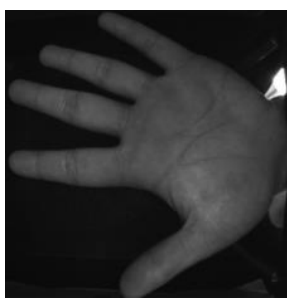

(a)

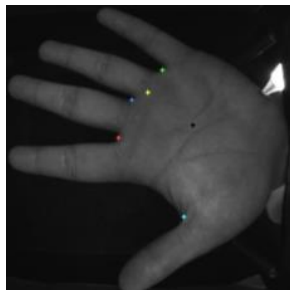

(d)

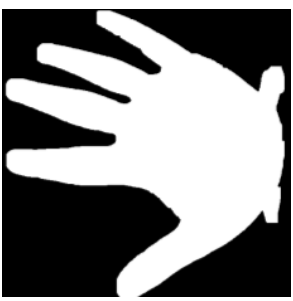

(b)

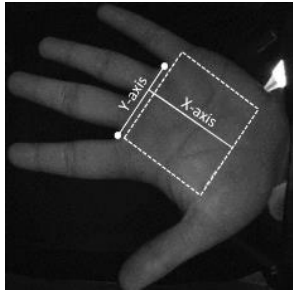

(e)

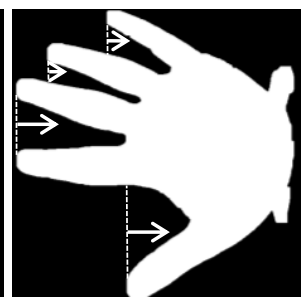

(c)

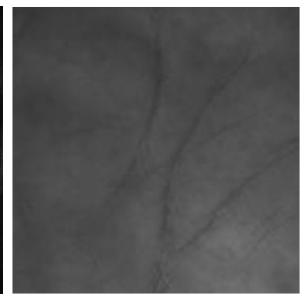

(f)
Fig. 2. Extraction of ROI from sample image. (a) Input image

(b) Pre-processed binary image (c) Starting search for mid-points (d) Overlay extracted landmarks and reference points on input image (e) Location of ROI (f) Extracted ROI.

\section{MULTISPECTRAL PALMPRINT IMAGE ANALYSIS}

\section{A. Directional Feature Encoding}

The undecimated dual tree complex wavelet transform (UDTCWT) [43] is an improved expansion of DTCWT. Two different form UDTCWT is presented in [43]. One of them is termed as U1DT-CWT, which provides exact shift invariance properties with the advantage of having one-to-one map in each subband with the same directional selectivity as DTCWT, since downsampling and upsampling of the filters are removed like a simple UDWT. Having a consistent size in all scales is an important relationship in the applications such as image denoising and image fusion. The second form of UDTCWT is termed as U2DT-CWT, which is retaining subsampling after the first level to reduce overcompleteness. However, sub-bands are not same size as input image in this form. In this paper, we use the shift invariance, one-to-one map in each subband and directional selectivity properties of the U1DT-CWT [43] for directional feature encoding in palmprint images.

A biorthogonal set of filters $\left(B_{f}\right)$ is used at the first scale to be convolved with an ROI $I \in \square^{m \times n}$. This step helps us to capture the details in the palm and allows robust information to be passed on to the subsequent stage.

$$
\rho=I * B_{f}
$$

The filtered component $(\rho)$ of the input image is processed by the q-step filters $\left(D_{f}\right)$ which comprise six complex directional subbands.

$$
\Psi^{i}=\rho^{*} D_{f}^{i}
$$

Where: $\Psi^{i}$ - indicates six set of directional complex subbands, $i=1,2, \ldots, 6$. The combination of biorthogonal and q-step filter decomposition stages lets us determine the 
capability of the system to capture line-like features. Usually, both the vein and line characteristics appear as black intensities in a palm. This corresponds to a negative filter response. The minimum peak response among all directional subbands is selected at a specific point. Assume $\Psi_{x, y}^{i}$ represents the coefficient at point $(x, y)$ in the $i^{\text {th }}$ directional subband where $i=1,2, \ldots, 6$. We use a similar rule to the competitive rule [38]. The dominant orientation in each $(x, y)$ is encoded.

$$
C=\arg _{i} \min \left(\Psi^{i}\right)
$$

where $C-$ denotes the U1DT-CWT coefficients that represents $I$. Note that $C$ is calculated for all subbands of the multispectral picture of a palm.

\section{B. Binary Encoding}

The binary encoding process presented in [42] is used to calculate one-to-many matching in palm image identification. The binary hash table method is applied to equation (3). The coefficients of the U1DT-CWT in equation (3) is then binarized. It should be noted that palmprint features are nonrigid, so it is not possible to have an exact 1:1 correspondence between all the directions of two palmprint pictures captured at different instances of time. Consequently, it is intuitive to set multiple orientations to a hash location $(x, y)$ based on its neighborhood. The blurring depends on a specific neighborhood rather than a single pixel and robustly catches crossover line orientations. Less blurring occurs in a few number of points matched with high confidence. Conversely, high-level blurring will result in large numbers being matched with low confidence.

The maximum score within all translations is defined as the final match. The class of inquiry palm is identified by the nth image in the gallery which corresponds to the highest match as defined in [42]:

$$
\text { Class }=\arg _{n} \max \left(S_{t_{x}, t_{y}}^{Q_{n}}\right)
$$

Here: $S_{t_{x}, t_{y}}^{Q_{n}}-$ is a match score. The bands are differently translated to determine the best possible combination [42]. In the matching process, each band is matched individually. The resulting scores are combined. Floating point comparisons are not needed for final decision, because a matching score is always an integer.

\section{EXPERIMENTAL RESULTS}

\section{A. Databases}

Two publicly available datasets are used for experiments: the CASIA [44] and PolyU [45]. Both datasets have low resolution (less than $150 \mathrm{dpi}$ ) multispectral palmprint images and that is stored as 8-bit images per band. ROIs of the PolyU database are already extracted by the method presented in [13].
2D Undecimated Dual Tree Complex Wavelets

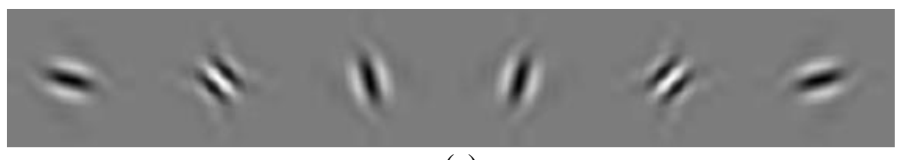

(a)

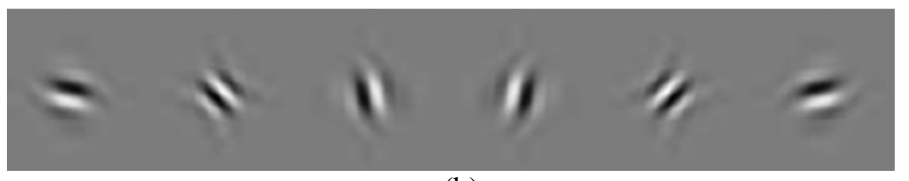

(b)

Fig. 3 Impulse response of 2D complex-valued wavelet filters. (a) Real part (b) Imaginary part.

The ROIs of the CASIA databases are not extracted and we apply landmark localization with ROI extraction strategy discussed in Section 3 in order to extract them. CASIA databases are captured by using a non-contact sensor and contains too many RST variations.

\section{B. Parameter Selection}

The effect of various parameters is studied such as ROI size, number of scales in the presented method, influence of binary encoding with blurring and non-blurring neighborhood. All parameters except the analysis of a specific parameter are kept fixed. This experimental analysis is carried out by using a sample set of the PolyU multispectral palmprint database compromising exact number of images from the first and second sessions. Optimal parameters found for PolyU databases are applied for the CASIA database.

\section{1) Dimension of ROI}

The length and thickness of the palmprint features vary. The most discriminative data can be lost with a small ROI. Moreover, unnecessary details and features can be included with a larger ROI. Different size of ROI regions with square sets such as $(16,16),(32,32), \ldots,(128,128)$ are empirically tested. Minimum EER is found at $(32,32)$. ROI size of $(32,32)$ is used in all our experiments.

\section{2) Scale Setting}

U1DT-CWT results in six complex directional subbands in the orientation of $\pm 15^{0}, \pm 45^{0}, \pm 75^{0}$. The six directional subbands for the impulse response of the filters are illustrated in Fig. 3. We consider only the real parts of the directional complex subbands, since the most promising feature for low-resolution image is in the real part. Different scale sizes are tested. Empirical tests show that scale 2 gives minimum EER. Therefore, two-scale decomposition is used in all experiments.

\section{3) Matching with Blurring Neighborhood}

The given palm is not a fixed object. Therefore, hash table blurring is applied to prevent minor misalignments. Moreover, too much blurring can result in false matches. The lowest ERR is obtained with four-adjoint blur neighborhood. 


\section{4) Filter Selection}

Biorthogonal sets of filters must be chosen for the first scale [43] and NDAntonB2 filter is used. In the remaining scales, qstep filters can be applied [43], [44]. NDdualfilt1 filter is used in the remaining scales.

\section{Validity of Experiments}

The PolyU and CASIA databases are used to perform the experiments. In both cases, half of the sample for each identity was obtained in one session and the other half was obtained in another. We used session-based Sets as discussed in [11] to observe recognition performance. We ran five experiments to evaluate the method in real life scenarios. The analyses of the experiments continued by matching:

Set 1: session regardless of the individual bands.

Set 2: palmprints (multispectral) from the first session.

Set 3: palmprints (multispectral) from the second session.

Set 4: palmprints (multispectral) of the first session to the second session.

Set 5: palmprints (multispectral) regardless of the session.

For the recognition experiments, the ROC curves are used to show variations between False Acceptance Rate (FAR) to the False Rejection Rate (FRR). The Genuine Acceptance Rate (GAR) at $0.1 \%$ FAR and the Equal Error Rate (EER) are selected to evaluate the recognition performance of the presented method. The presented method is compared with three state-of-the-art techniques: CompCode, DoGRepr and OrdRepr. We use our implementation for these three techniques since their code is not public. Table 1 gives the number of genuine and imposter matches for each sets. We apply four-adjoint blur neighborhood for gallery binary hash table encoding. Furthermore, the matching process is carried out by score-level band fusion.

TABLE I
GENUINE AND IMPOSTER MATCHES CONSIDERED IN
EACH SET.
\begin{tabular}{|c|c|c|c|c|}
\hline \multirow{3}{*}{} & \multicolumn{4}{|c|}{ Experiments } \\
\cline { 2 - 5 } & & Set $\mathbf{2} \mathbf{3}$ & Set $\mathbf{4}$ & Set $\mathbf{1} \mathbf{5}$ \\
\hline \multirow{3}{*}{ CASIA } & Gen. & 600 & 1,800 & 3,000 \\
& Imp. & 179,100 & 358,200 & 716,400 \\
\hline \multirow{3}{*}{ PolyU } & Gen. & 7,500 & 18,000 & 33,000 \\
& Imp. & $4,491,000$ & $8,982,000$ & $17,964,000$ \\
\hline
\end{tabular}

\section{1) Set 1}

This set analyzes the discriminant capability of individual bands as depicted in Fig. 4. Table 2 compares individual bands of palm performance of the PolyU and CASIA database for the presented code with blurring and non-blurring case. For the PolyU database, the $660 \mathrm{~nm}$ band gives the best performance for blurring and non-blurring. A major reason for this could be that the $660 \mathrm{~nm}$ wavelength mostly catches both the line and vein features which make this band more
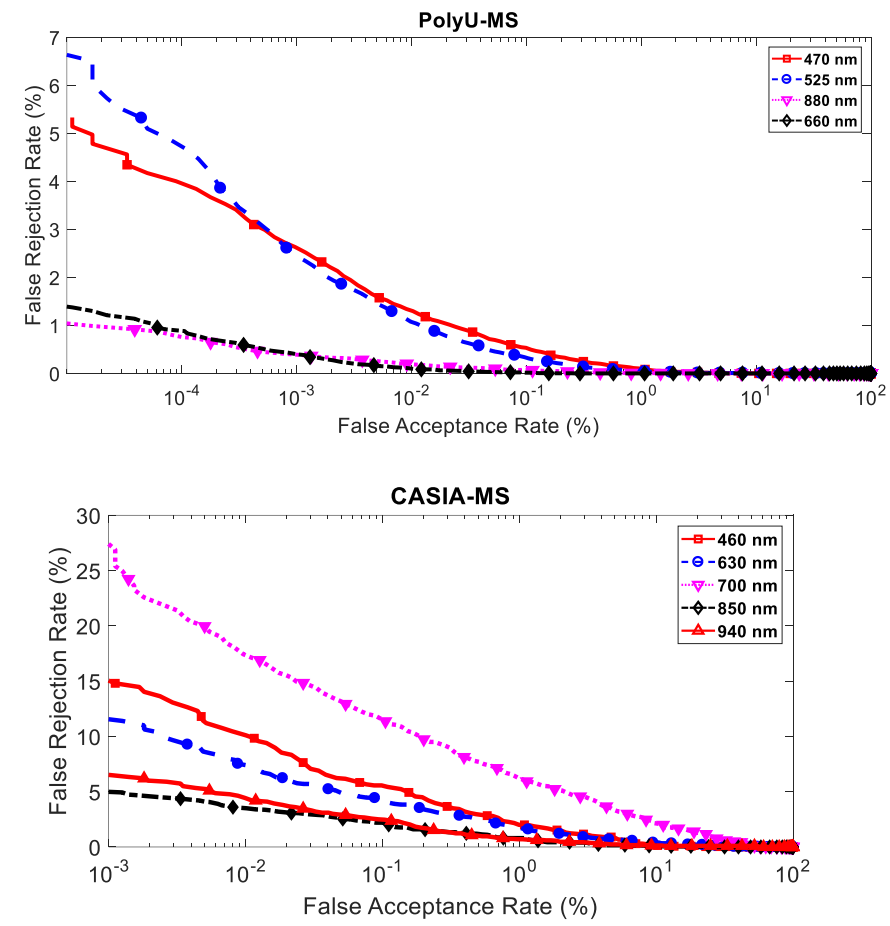

Fig. 4. Individual bands considered with blurring effect for PolyU and CASIA databases by proposed method in Set 1 .

TABLE II

PERFORMANCE OF THE INDIVIDUAL BANDS FOR THE PROPOSED METHOD.

\begin{tabular}{|c|c|c|c|c|c|c|}
\hline \multirow[b]{2}{*}{ 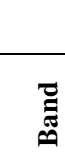 } & & \multicolumn{2}{|c|}{ PolyU } & \multicolumn{3}{|c|}{ CASIA } \\
\hline & & Blur & $\begin{array}{l}\text { Non- } \\
\text { blur }\end{array}$ & صี & Blur & $\begin{array}{l}\text { Non- } \\
\text { blur }\end{array}$ \\
\hline $\begin{array}{l}470 \\
\mathrm{~nm}\end{array}$ & $\begin{array}{l}\text { GAR }(\%) \\
\operatorname{EER}(\%)\end{array}$ & $\begin{array}{c}99.47 \\
0.2766\end{array}$ & $\begin{array}{c}99.07 \\
0.3820\end{array}$ & $\begin{array}{l}460 \\
\mathrm{~nm}\end{array}$ & $\begin{array}{c}94.45 \\
1.6484\end{array}$ & $\begin{array}{c}93.64 \\
1.7821\end{array}$ \\
\hline $\begin{array}{l}525 \\
\mathrm{~nm}\end{array}$ & $\begin{array}{l}\operatorname{GAR}(\%) \\
\operatorname{EER}(\%)\end{array}$ & $\begin{array}{c}99.67 \\
0.2028\end{array}$ & $\begin{array}{c}99.35 \\
0.2880\end{array}$ & $\begin{array}{l}630 \\
\mathrm{~nm}\end{array}$ & $\begin{array}{c}95.84 \\
1.3907\end{array}$ & $\begin{array}{c}96.09 \\
1.3628\end{array}$ \\
\hline $\begin{array}{l}660 \\
\mathrm{~nm}\end{array}$ & $\begin{array}{l}\operatorname{GAR}(\%) \\
\operatorname{EER}(\%)\end{array}$ & $\begin{array}{c}99.98 \\
0.0381\end{array}$ & $\begin{array}{c}99.93 \\
0.0842\end{array}$ & $\begin{array}{l}700 \\
\mathrm{~nm}\end{array}$ & $\begin{array}{c}87.48 \\
3.8757\end{array}$ & $\begin{array}{c}90.83 \\
2.8488\end{array}$ \\
\hline $\begin{array}{l}880 \\
\mathrm{~nm}\end{array}$ & $\begin{array}{l}\operatorname{GAR}(\%) \\
\operatorname{EER}(\%)\end{array}$ & $\begin{array}{c}99.93 \\
0.0754\end{array}$ & $\begin{array}{c}99.88 \\
0.1118\end{array}$ & $\begin{array}{l}850 \\
\mathrm{~nm}\end{array}$ & $\begin{array}{c}97.80 \\
0.8000\end{array}$ & $\begin{array}{l}98.30 \\
0.6427\end{array}$ \\
\hline & $\begin{array}{l}\text { GAR \%) } \\
\operatorname{EER}(\%)\end{array}$ & & & $\begin{array}{l}940 \\
\mathrm{~nm}\end{array}$ & $\begin{array}{c}97.52 \\
0.7642\end{array}$ & $\begin{array}{c}97.90 \\
0.6538\end{array}$ \\
\hline
\end{tabular}

discriminative. For the CASIA database, the most discriminant data is available in the $850 \mathrm{~nm}$ and $940 \mathrm{~nm}$ bands which give the best results with and without consideration of the blurring effect.

\section{2) $\operatorname{Set} 2$}

Set 2 examines the variability in the palmprint information obtained in the first session. Fig. 5 analyzes the ROC curves of the proposed method with three techniques on the CASIA and PolyU databases. 

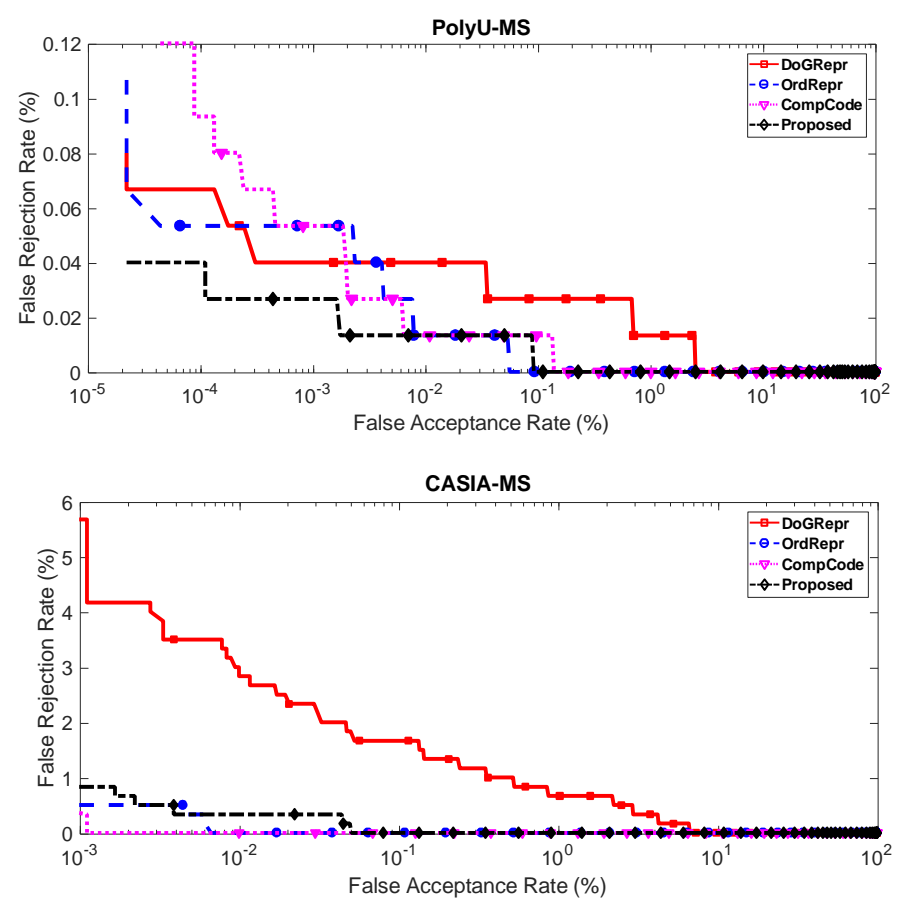

Fig. 5. Multispectral palmprints obtained in first session with blurring effect for PolyU and CASIA databases in Set 2.
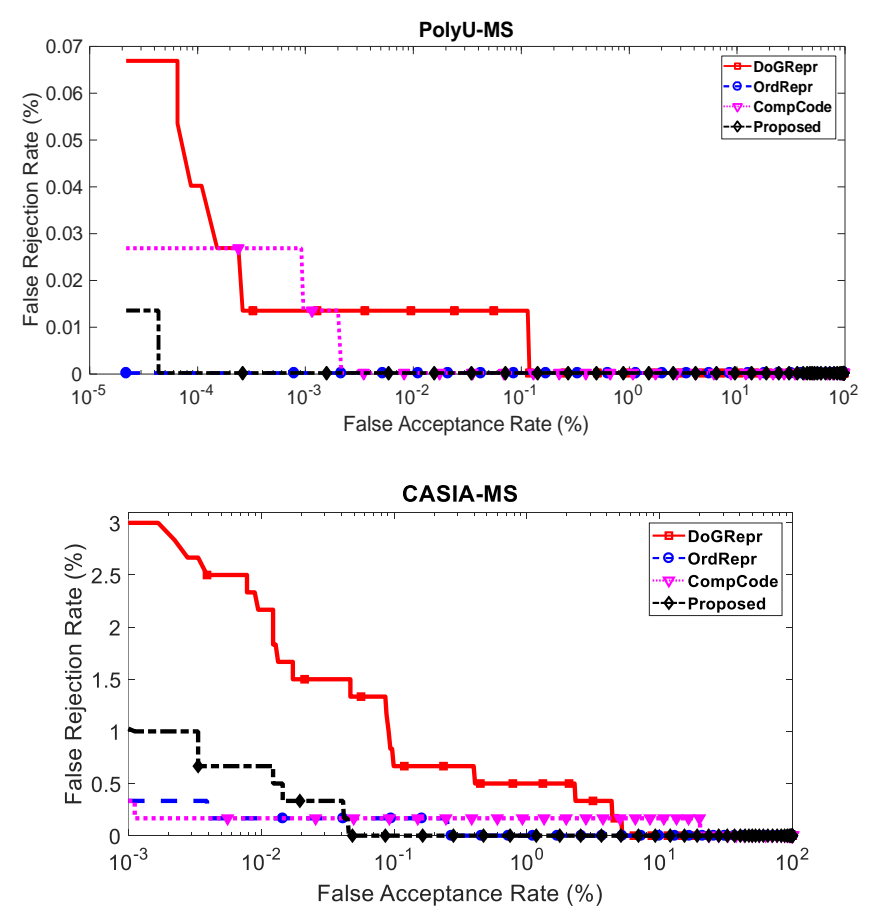

Fig. 6. Multispectral palmprints gained in second session with blurring effect for PolyU and CASIA.

3) Set 3

Set 3 investigates the variability in the palmprint information gained in the second session as depicted in Fig. 6. In this setwe investigate the intra-band variations compare the results obtained in Set 2. Furthermore, only the palmprints obtained in the second session are matched.
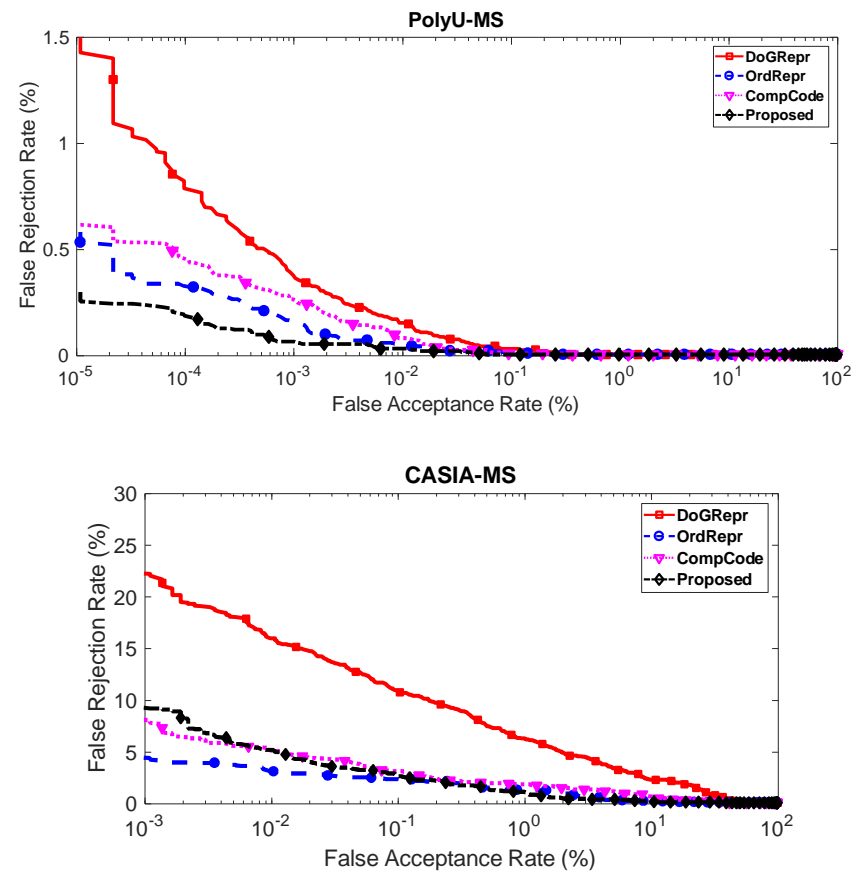

Fig. 7. Multispectral palmprints of the first session to the second session with blurring effect for PolyU and CASIA databases in Set 4.
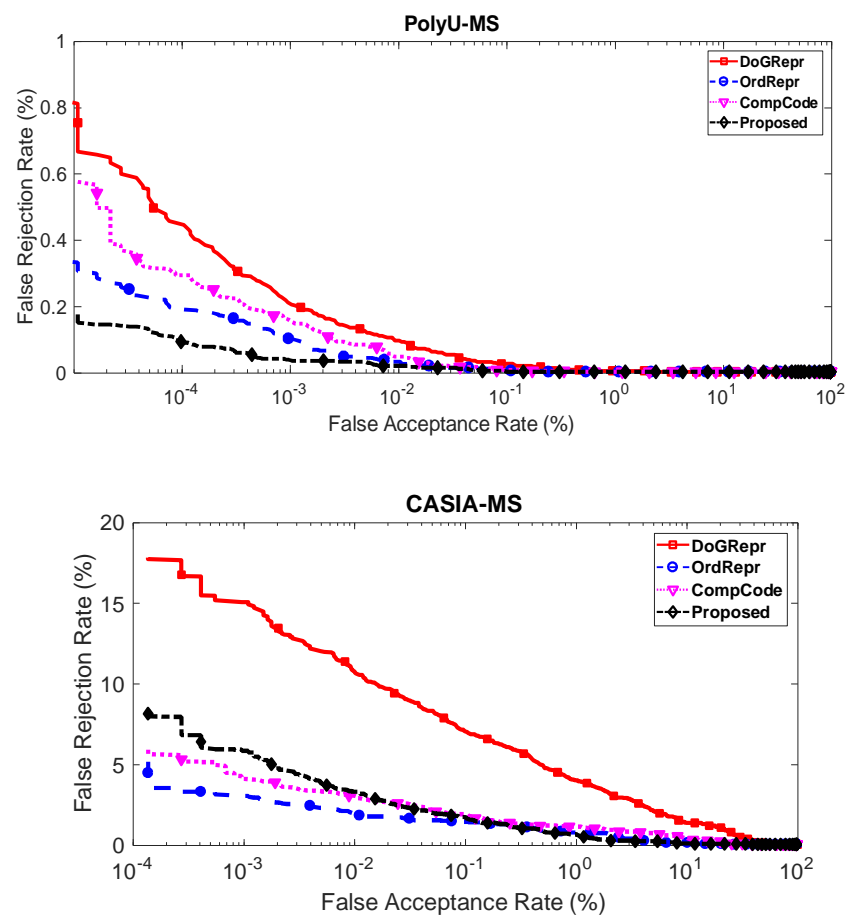

Fig. 8. Multispectral palmprints regardless of the session with blurring effect for PolyU and CASIA databases in Set 5.

4) $\operatorname{Set} 4$

Set 4 is used to test a real-life scenario where there is a variation in image quality and/or human behavior over time. According to this, all images from the first session are matched to all images of the second. Fig. 7 compares the ROC curves of the proposed code with three methods. It should be mentioned that there is a performance degeneration of other methods compared to Set 2 and Set 3 because of the difficulty 
TABLE III

VERIFICATION EXPERIMENTS FOR SET 2 TO SET 5 WITH BLURRING.

\begin{tabular}{|c|c|c|c|c|c|}
\hline & \multicolumn{5}{|c|}{ CASIA Database } \\
\hline Methods & & Set 2 & Set 3 & Set 4 & Set 5 \\
\hline \multirow{2}{*}{ DoGRepr } & $\operatorname{EER}(\%)$ & 0.8333 & 0.5000 & 3.8889 & 2.8667 \\
\hline & GAR (\%) & 98.33 & 99.33 & 89.18 & 92.97 \\
\hline \multirow{2}{*}{ OrdRepr } & $\operatorname{EER}(\%)$ & 0.0067 & 0.1667 & 1.2222 & 0.7667 \\
\hline & GAR $(\%)$ & 100.00 & 98.83 & 97.72 & 98.60 \\
\hline \multirow{2}{*}{ CompCode } & $\operatorname{EER}(\%)$ & 0.0011 & 0.1667 & 1.6111 & 1.1000 \\
\hline & GAR $(\%)$ & 100.00 & 98.83 & 96.94 & 98.13 \\
\hline \multirow{3}{*}{ Proposed } & $\operatorname{EER}(\%)$ & 0.0512 & 0.0455 & 1.0154 & 0.6874 \\
\hline & GAR $(\%)$ & 100.00 & 100.00 & 97.42 & 98.30 \\
\hline & \multicolumn{5}{|c|}{ PolyU Database } \\
\hline Methods & & Set 2 & Set 3 & Set 4 & Set 5 \\
\hline \multirow{2}{*}{ DoGRepr } & $\operatorname{EER}(\%)$ & 0.0353 & 0.0134 & 0.0462 & 0.0402 \\
\hline & GAR $(\%)$ & 99.97 & 99.99 & 99.97 & 99.98 \\
\hline \multirow{2}{*}{ OrdRepr } & $\operatorname{EER}(\%)$ & 0.0134 & 0.000 & 0.0208 & 0.0182 \\
\hline & $\operatorname{GAR}(\%)$ & 100.00 & 100.00 & 99.99 & 100.00 \\
\hline \multirow{2}{*}{ CompCode } & $\operatorname{EER}(\%)$ & 0.0134 & 0.0022 & 0.0278 & 0.0212 \\
\hline & GAR $(\%)$ & 99.99 & 100.00 & 99.99 & 99.99 \\
\hline \multirow{2}{*}{ Proposed } & $\operatorname{EER}(\%)$ & 0.0134 & 0.0000 & 0.0167 & 0.0148 \\
\hline & $\operatorname{GAR}(\%)$ & 100.00 & 100.00 & 100.00 & 100.00 \\
\hline
\end{tabular}

TABLE IV

VERIFICATION EXPERIMENTS FOR SET 2 TO SET 5 WITH NON-BLURRING.

\begin{tabular}{|c|c|c|c|c|c|}
\hline & \multicolumn{5}{|c|}{ CASIA Database } \\
\hline Methods & & Set 2 & Set 3 & Set 4 & Set 5 \\
\hline DoGRepr & $\begin{array}{l}\operatorname{EER}(\%) \\
\operatorname{GAR}(\%)\end{array}$ & $\begin{array}{c}0.3225 \\
99.00\end{array}$ & $\begin{array}{c}0.3333 \\
99.67\end{array}$ & $\begin{array}{c}2.4444 \\
93.22\end{array}$ & $\begin{array}{c}1.6813 \\
95.67\end{array}$ \\
\hline OrdRepr & $\begin{array}{l}\operatorname{EER}(\%) \\
\operatorname{GAR}(\%)\end{array}$ & $\begin{array}{l}0.0234 \\
100.00\end{array}$ & $\begin{array}{c}0.1667 \\
99.83\end{array}$ & $\begin{array}{c}0.7015 \\
98.22\end{array}$ & $\begin{array}{c}0.5333 \\
98.90\end{array}$ \\
\hline CompCode & $\begin{array}{l}\operatorname{EER}(\%) \\
\operatorname{GAR}(\%)\end{array}$ & $\begin{array}{l}0.0295 \\
100.00\end{array}$ & $\begin{array}{c}0.1667 \\
99.83\end{array}$ & $\begin{array}{c}1.3000 \\
97.17\end{array}$ & $\begin{array}{c}0.8667 \\
98.27\end{array}$ \\
\hline Proposed & $\begin{array}{l}\operatorname{EER}(\%) \\
\operatorname{GAR}(\%)\end{array}$ & $\begin{array}{l}0.0926 \\
100.00\end{array}$ & $\begin{array}{c}0.1667 \\
99.83\end{array}$ & $\begin{array}{c}0.8889 \\
97.44\end{array}$ & $\begin{array}{c}0.7262 \\
98.43\end{array}$ \\
\hline & \multicolumn{5}{|c|}{ PolyU Database } \\
\hline Methods & & Set 2 & Set 3 & Set 4 & Set 5 \\
\hline DoGRepr & $\begin{array}{l}\operatorname{EER}(\%) \\
\operatorname{GAR}(\%)\end{array}$ & $\begin{array}{l}0.0267 \\
100.00\end{array}$ & $\begin{array}{l}0.0025 \\
100.00\end{array}$ & $\begin{array}{l}0.0278 \\
100.00\end{array}$ & $\begin{array}{l}0.0242 \\
100.00\end{array}$ \\
\hline OrdRepr & $\begin{array}{l}\operatorname{EER}(\%) \\
\operatorname{GAR}(\%)\end{array}$ & $\begin{array}{c}0.0400 \\
99.97\end{array}$ & $\begin{array}{l}0.0000 \\
100.00\end{array}$ & $\begin{array}{c}0.0225 \\
99.98\end{array}$ & $\begin{array}{c}0.0221 \\
99.98\end{array}$ \\
\hline CompCode & $\begin{array}{l}\operatorname{EER}(\%) \\
\operatorname{GAR}(\%)\end{array}$ & $\begin{array}{c}0.0267 \\
99.99\end{array}$ & $\begin{array}{l}0.0006 \\
100.00\end{array}$ & $\begin{array}{l}0.0207 \\
100.00\end{array}$ & $\begin{array}{l}0.0182 \\
100.00\end{array}$ \\
\hline Proposed & $\begin{array}{l}\operatorname{EER}(\%) \\
\operatorname{GAR}(\%)\end{array}$ & $\begin{array}{c}0.0359 \\
99.99\end{array}$ & $\begin{array}{l}0.0013 \\
100.00\end{array}$ & $\begin{array}{c}0.0500 \\
99.97\end{array}$ & $\begin{array}{c}0.0364 \\
99.98\end{array}$ \\
\hline
\end{tabular}

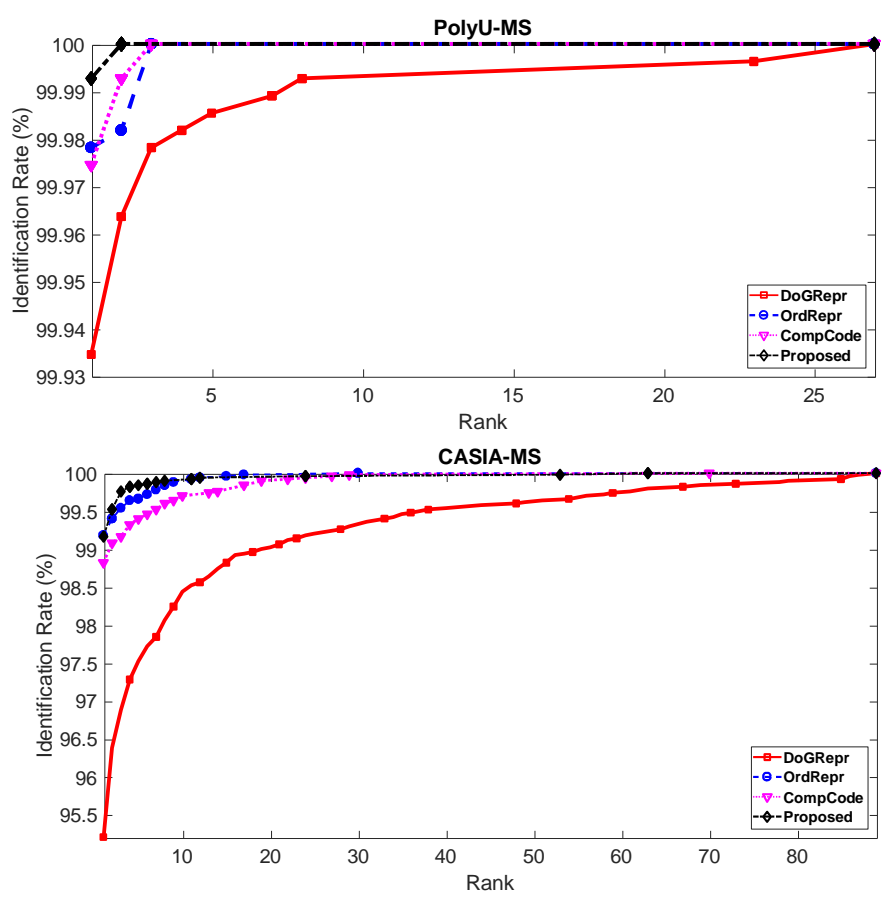

Fig. 9. Identification performance with CMC curves.

of the scenario. However, degeneration of the proposed method is much less than of other methods on PolyU database, which indicates the robustness of the proposed method to the image variability.

\section{5) Set 5}

Set 5 analyzes the overall recognition performance which allows comparison of existing methods with the proposed method on the same database. This set is termed to be "all versus all" in the literature since all images in that database are compared to all other images. The proposed method is compared with three other methods in Fig. 8 considering this scenario. The proposed method outperforms all other methods on the PolyU database and gives comparable results on the CASIA database.

\section{Comparison with State-of-the-Art Methods}

The results obtained in Set 2 to Set 5 are given in Table 3. The proposed method gives the best results in the PolyU database compared to the three state-of-the-art methods for blurring case. Furthermore, the proposed method outperforms in some sets considering the CASIA database. It should be noted that OrdRepr has more comparable results than the other methods for blurring case on the CASIA database. In Table 4, the propose method still gives a very good comparable result although it might not give the best results in some cases compared to the other state-of-the-art methods in the CASIA database. Since the palm images were acquired with the use of contact-based sensors in the PolyU database, all methods produce better EER performance. The EER performance of CompCode and DoGRepr deteriorated and produce the worst results of all other methods in the CASIA database with blurring. At the same time, the presented method gives much 
better EER performance with blurring.

\section{E. Validity of Experiments}

Identification experiments are performed by using fivefold cross validation and identification rates at rank-1 and reported with the Cumulative Match Characteristics (CMC) curves. We randomly choose one multispectral palm image per subject to set the gallery and all other images as test samples in each fold. In this way, a single multispectral palm image in the gallery is used for identification for any test sample subject. Then, the average identification rates are computed over fivefold. We use this protocol for both databases. Fig. 9 gives the CMC curves to compare the performance of the proposed method with other three state-of-the-art methods with blurring effect. The identification performances are given in Table 5 and Table 6 for the blurring and non-blurring case, respectively. Both tables indicate that the proposed method gives the best and results compared to the three state-of-the-art methods.

\section{CONCLUSION}

This paper presents a new multidirectional and multiscale analysis based on UDTCWT for feature encoding by applying a binary hash table matching procedure for palm image recognition. Newly, a simple DWT is also used to extract the ROIs rather than the usual method that uses bicubic interpolation to reduce noisy regions and inconsistent lines. With this approach, the performance of the three state-of-theart methods are compared. The proposed multidirectional and multiscaling feature extraction method provides robust and effective palmprint representation and matching compared to three of the state-of-the-art methods. Furthermore, the matching speed is increased by using the score-level-fusion method for effective palmprint recognition. Results obtained in the experimental part show that the proposed method gives the best results for blurring case and comparable results for non-blurring case when compared with the state-of-the-art methods. UDT-CWT based feature encoding can be easily applied to line-like features and can be extended to other types of biometrics.

\section{REFERENCES}

[1] D. D. Zhang, Automated Biometrics-Technologies and Systems, Boston: Kluwer Academic Publishers, pp. 3-18, 2000.

[2] T. Connie, A. Jin, M. Ong, D. Ling, "An automated palmprint recognition system”, Image Vis. Computation., vol. 23, no. 5, pp. 501505, May 2005.

[3] A. Kong, D. Zhang, M. Kamel, "Palmprint identification using featurelevel fusion", Pattern Recog., vol. 39, no. 3, pp. 478-487, Mar. 2006.

[4] A. Kong, D. Zhang, "Competitive coding scheme for palmprint verification", in Proc. Int. Conf. Pattern Recog., 2004, pp. 520-523.

[5] W. Jia, D. Huang, D. Zhang, "Palmprint verification based on robust line orientation code", Pattern Recog., vol. 41, no. 5, pp. 1504-1513, May 2008.

[6] A. Kumar, D. Zhang, "Personal recognition using hand shape and texture", IEEE Trans. Image Process., vol. 15, no. 8, pp. 2454-2461, Aug. 2006.

TABLE V
CORRELATION OF RANK-1 RECOGNITION RATES AND STANDARD DEVIATIONS ON THE CASIA AND POLYU DATABASES FOR BLURRING. IDENTIFICATION RATES ARE AVERAGED OVER 5-FOLD.

\begin{tabular}{|c|c|c|}
\hline Methods & CASIA (\%) & PolyU (\%) \\
\hline DoGRepr & $95.20 \pm 0.81$ & $99.98 \pm 0.02$ \\
\hline OrdRepr & $99.18 \pm 0.15$ & $100.00 \pm 0.01$ \\
\hline CompCode & $98.82 \pm 0.22$ & $99.99 \pm 0.02$ \\
\hline Proposed & $99.16 \pm 0.26$ & $100.00 \pm 0.01$ \\
\hline
\end{tabular}

TABLE VI

CORRELATION OF RANK-1 RECOGNITION RATES AND STANDARD DEVIATIONS ON THE CASIA AND POLYU DATABASES FOR NON-BLURRING. IDENTIFICATION RATES ARE AVERAGED OVER 5-FOLD

\begin{tabular}{|c|c|c|}
\hline Methods & CASIA (\%) & PolyU (\%) \\
\hline DoGRepr & $97.46 \pm 0.34$ & $99.95 \pm 0.07$ \\
\hline OrdRepr & $99.60 \pm 0.25$ & $99.99 \pm 0.02$ \\
\hline CompCode & $99.04 \pm 0.36$ & $99.99 \pm 0.01$ \\
\hline Proposed & $99.30 \pm 0.19$ & $99.97 \pm 0.04$ \\
\hline
\end{tabular}

[7] S. Schuckers, "Spoofing and anti-spoofing measures", Inf. Secure. Tech. Rep., vol. 7, no. 4, pp. 56-62, Dec. 2002.

[8] R. Rowe, K. Nixon, S. Corcoran, "Multi spectral fingerprint biometrics", in Proc. Inf. Assurance Workshop, 2005, pp. 14-20.

[9] J. Park, M. Kang, "Multispectral iris authentication system against counterfeit attack using gradient-based image fusion”, Opt. Eng., vol. 46, no. 11, pp. 117003-1-117003-14, Nov. 2007.

[10] R. Singh, M. Vatsa, A. Noore, "Hierarchical fusion of multispectral face images for improved recognition performance", Inf. Fusion, vol. 9, no. 2, pp. 200-210, Apr. 2008.

[11] D. Zhang, W. Zuo, F. Yue, "A comparative study of palmprint recognition algorithms", ACM Computing Surveys (CSUR), vol. 44, no. 1, pp. 2:1-2:37, Jan. 2012.

[12] A. Jain, J. Feng, "Latent palmprint matching", IEEE Trans. on Pattern Anal. Mach. Intell., vol. 31, no. 6, pp. 1032-1047, Oct. 2009.

[13] D. Zhang, W. Kong, J. You, M. Wong, "Online palmprint identification", IEEE Trans. on Pattern Anal. Mach. Intell., vol. 25, no. 9, pp. 1041-1050, Sept. 2003.

[14] D. Han, Z. Guo, D. Zhang, "Multispectral palmprint recognition using wavelet-based image fusion", in Proc. Int. Conf. on Signal Processing, 2008, pp. 2074-2077.

[15] Y. Hao, Z. Sun, T. Tanet, "Comparative studies on multispectral palm image fusion for biometrics", in Proc. Asian Conf. on Computer Vision, Springer, pp. 12-21, 2007.

[16] A. Jain, A. Ross, S. Prabhakar, "An introduction to biometric recognition", IEEE Trans. on Circuits and Systems for Video Technology, vol. 14, no. 1, pp. 4-20, Jan. 2004.

[17] C. Boyce, A. Ross, M. Monaco, L. Hornak, X. Li., "Multispectral iris analysis: A preliminary study", in Proc. Computer Vision and Pattern Recognition Workshops (CVPRW), July 2006.

[18] W. Di, L. Zhang, D. Zhang, Q. Pan, "Studies on hyperspectral face recognition in visible spectrum with feature band selection", IEEE Transactions on Systems, Man, and Cybernetics - Part A: Systems and Humans, vol. 40, no. 6, pp. 1354-1361, 2010.

[19] Z. Pan, G. Healey, M. Prasad, B. Tromberg, "Face recognition in hyperspectral images," IEEE Trans. on Pattern Anal. Machine Intelligence, vol. 25, no. 12, pp. 1552-1560, Dec. 2003.

[20] R. Rowe, U. Uludag, M. Demirkus, S. Parthasaradhi, A. Jain, "A multispectral whole-hand biometric authentication system", in IEEE Biometrics Symposium, 2007, pp. 1-6. 
[21] A. Meraoumia, S. Chitroub, A. Bouridane, "An efficient palmprint identification system using multispectral and hyperspectral imaging", in Modeling Approaches and Algorithms for Advanced Computer Applications, vol. 488, pp. 155-164, 2013.

[22] M. Bounneche, L. Boubchir, A. Bouridane, B. Nekhoul, and A. AliCherif, "Multi-spectral palmprint recognition based on oriented multiscale log-Gabor filters", Neurocomputing, vol. 205, pp. 274-286, Sept. 2016.

[23] N. Luo, Z. Gang, W. C. Song, "Multispectral palmprint recognition by feature level fusion", in Recent Advances in Computer Science and Inf. Engineering, Springer, vol. 128, pp. 427-432, 2012.

[24] S. Mistani, S. Minaee, E. Fatemizadeh, "Multispectral palmprint recognition using a hybrid feature", vol. abs/1112.5997, Dec. 2011.

[25] Y. Aberni, L. Boubchir, B. Daachi, "Multispectral palmprint recognition: A state-of-the-art review", in Int. Conf. on on Telecommunications and Signal Processing (TSP), 2017, pp. 793-798.

[26] A. Tahmasebi, H. Pourghasem, H. Mahdavi-Nasab, "A novel rank level fusion for multispectral palmprint identification system", in Proc. Int. Conf. on Intelligent Computation and Bio-Medical Instr. (ICBMI), 2011, pp. 208-211.

[27] D. Zhang, Z. Guo, G. Lu, L. Zhang, Y. Liu, W. Zuo, "Online joint palmprint and palmvein verification", Expert Systems with Applications, vol. 38, no. 3, pp. 2621-2631, March 2011.

[28] D. Kisku, P. Gupta, J. Sing, C. Hwang, "Multispectral palm image fusion for person authentication using ant colony optimization", in Proc. Int. Works. on Emerging Tech. and Challenges for Hand-Based Biometrics (ETCHB), 2010, pp. 1-7.

[29] Z. Guo, D. Zhang, L. Zhang, W. Liu, "Feature band selection for multispectral palmprint recognition", in Proc. Int. Conf. on Pattern Recog. (ICPR), 2010, pp. 1136-1139.

[30] D. Zhang, Z. Guo, G. Lu, L. Zhang, W. Zuo, "An online system of multispectral palmprint verification", IEEE Trans. on Instrumentation and Measurement, vol. 59, no. 2, pp. 480-490, Feb. 2010.

[31] X. Xu, Z. Guo, "Multispectral palmprint recognition using quaternion principal component analysis", in Proc. Int. Workshop on Emerging Tech. and Challenges for Hand-Based Biometrics (ETCHB), 2010, pp. $1-5$.

[32] Y. Hao, Z. Sun, T. Tan, C. Ren, "Multispectral palm image fusion for accurate contact-free palmprint recognition", in Proc. Int. Conf. on Image Process., 2008, pp. 281-284.

[33] A. Kong, D. Zhang, M. Kamel, "A survey of palmprint recognition", Pattern Recog., vol. 42, no. 7, pp. 1408-1418, July 2009.

[34] D. Huang, W. Jia, D. Zhang, "Palmprint verification based on principal lines", Pattern Recog., vol. 41, no. 4, pp. 1316-1328, April 2008.

[35] G. Lu, D. Zhang, K. Wanga, "Palmprint recognition using eigenpalms features", Pattern Recog. Letters, vol. 24, no. 9, pp. 1463-1467, June 2003.

[36] X. Wu, D. Zhang, K. Wanga, "Fisherpalms based palmprint recognition", Pattern Recog. Letters, vol. 24, no. 15, pp. 2829-2838, Nov. 2003

[37] J. Wang, W. Yau, A. Suwandy, E. Sung, "Fusion of palmprint and palm vein images for person recognition based on laplacian palm feature", in Proc. Comp. Vision and Pattern Recog., 2007, pp. 1-8.

[38] A.W.-K. Kong, D. Zhang, "Competitive coding scheme for palmprint verification", in Proc. Int. Conf. on Pattern Recog., pp. 520-523.

[39] Z. Sun, T. Tan, Y. Wang, S. Z. Li, "Ordinal palmprint representation for personal identification", in Proc. Computer Vision and Pattern Recog., 2005, pp. 279-284.

[40] X. Wu, K. Wang, D. Zhang, "Palmprint texture analysis using derivative of Gaussian filters", in Proc. Int. Conf. on Computational Intelligence and Security, 2006, pp. 751-754.

[41] Y. Zhou, A. Kumar, "Contactless palm vein identification using multiple representations", in Proc. Int. Conf. on Biometrics: Theory Applications and Systems (BTAS), 2010, pp. 1-6.

[42] Z. Khan, A. Mian, Y. Hu, "Contour code: Robust and efficient multispectral palmprint encoding for human recognition", in Proc. Int. Conf. on Computer Vision (ICCV), 2011, pp. 1935-1942.

[43] P. R. Hill, N. Anantrasirichai, A. Achim, M. E. Al-Mualla, D. R. Bulla, "Undecimated Dual-Tree Complex Wavelet Transforms", Signal Processing: Image Communication, vol. 35, pp. 61-70, July 2015.

[44] "CASIA Multispectral Palmprint Database." [Online]. Available: http://biometrics.idealtest.org, accessed Oct. 2018.

[45] "PolyU Multispectral Palmprint Database." [Online]. Available:https://www4.comp.polyu.edu.hk/ biometrics /MultispectralPalmprint/MSP.htm, accessed Oct. 2018.
[46] I. W. Selesnick, R. G. Baraniuk, N. C. Kingsbury, "The dual tree complex wavelet transform", IEEE Signal Processing Magazine, vol. 22, no. 6, pp. 123-151, Nov. 2005.

\section{BIOGRAPHIES}

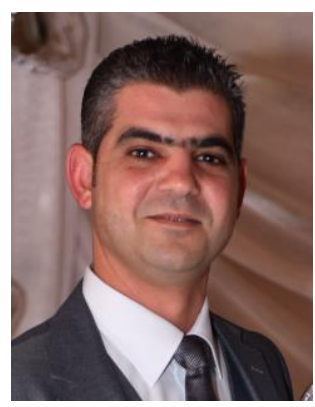

BURCIN OZMEN received his BSc. degree in Electrical and Electronics Engineering from Eastern Mediterranean University, Gazimagusa, North Cyprus, in 1999, the M.Sc. degree in Electrical and Electronic Engineering from Eastern Mediterranean University, Gazimagusa, North Cyprus, in 2001. Ozmen was awarded the PhD degree in 2007 for his work in the area of signal processing. Presently, he is working as an Assistant Professor in European University of Lefke, Lefke, Northern Cyprus. His research interests include signal and image processing, computer vision, and sparse representation.

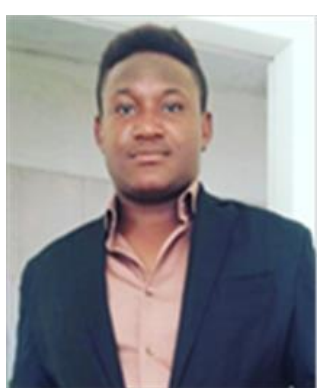

OLAYINKA JOHN OLALEYE received MSc. degree in Electrical and Electronics Engineering from the Cyprus International University, Lefkoşa, North Cyprus, in the year 2016. Presently, he is pursuing his PhD. in Electrical and Electronics Engineering Department of the Cyprus International University. 